\title{
Extraction of green fluorescent protein labelled Mesenchymal Stem Cells Imperial College to investigate their mechanisms of action on Ischemia- Reperfusion Injury London in a rat kidney transplant model.
}

N.Vallant ${ }^{1,2}$, J.Behmoaras ${ }^{1}$, J.Ko${ }^{1}$, K.Woollard ${ }^{1}$, A.Garcia-Diaz ${ }^{1}$, T.Page ${ }^{1}$, F.W.K.Tam ${ }^{1}$, B.Sandhu ${ }^{1}$, C.Pusey ${ }^{1}$, V. Papalois ${ }^{1}$ 1 Renal and Transplant Department, Imperial College London

2 Department of Visceral-, Transplant- and Thoracic Surgery, Medical University Innsbruck

Background: Ex vivo machine perfusion (MP) in solid organ transplantation is a promising tool for assessing and reconditioning suboptimal grafts by infusion of drugs or cells, such as mesenchymal stem cells (MSCs) ${ }^{1}$. MSCs are multipotent, self renewing cells with immunomodulatory and antiinflammatory capacities which have been suggested to home to sites of injury and promote regeneration ${ }^{2}$. However, in order to further investigate the effects of MSC infusion on an injured organ in a rodent kidney transplant model, delivered MSCs have to be distinguished from preexisting intragraft MSCs. The aim of this study was to investigate whether MSCs extracted from the bone marrow of transgenic Wistar Kyoto (WKY) rats positive for green fluorescent protein (GFP) are green upon correct wavelength excitation and if this was maintained during higher passages of the cell line. Furthermore, we wanted to investigate the immunomodulatory capacities of the GFP positive cells in different passages and directly compare them to MSCs extracted from WKY wildtype animals. Thereby, we hoped to reveal the ,ideal' traceable MSC type for application in the above mentioned model.

Methods: MSCs were extracted from femurs and tibias of WKY wildtype and GFP transgenic rats. Cells were cultured from passage 0-10 and supernatants as well as cells from each passage were frozen for future perfusion experiments. The MSC phenotype was confirmed by plastic adherence of the cells, flow cytometry and differentiation of the cells into adipocytes and osteocytes. Up to passage 10 the cells were observed for the presence of GFP using a confocal microscope. To investigate on immunomodulatory effects of MSCs from different animals (WT vs. GFP+, young versus old donors) and at different passages (P1-10), macrophages after activation with LPS were treated with respective supernatants over night and their expression of mannose-receptor 1 (Mrc1) as an indicator for transformation from M1 into M2 macrophages were compared by RT-qPCR. Statistical analysis was performed using wilcoxon-test.

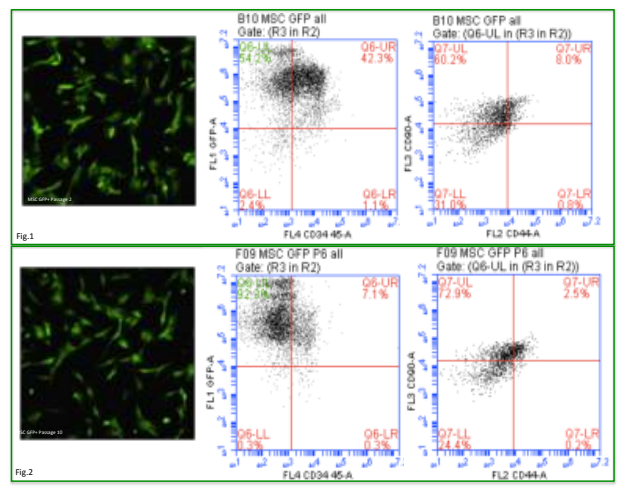

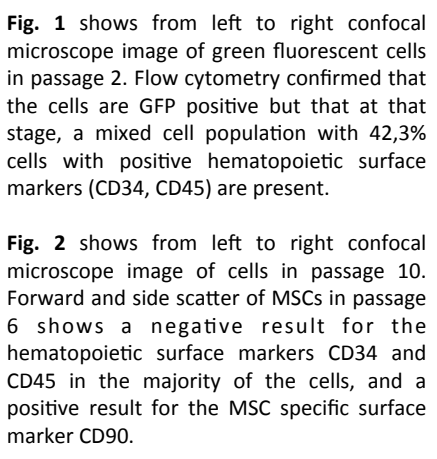

Fig. 1 shows from left to right confocal me of green fluorescent cells the cells are GFP positive but that at that stage, a mixed cell population with $42,3 \%$ cells with positive hematopoietic surface 2 shows from left to right confocal microscope image of cells in passage 10. shows a negative result for the hematopoietic surface markers CD34 and positive result for the MSC specific surface marker CD90.

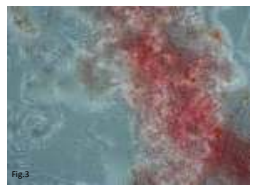

Fig. 3: Alizarin Red staining of GFP+ MSC in passage 5 after differentiation into osteocytes.

Alizarin Red staining

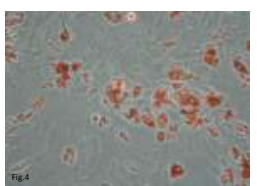

Fig. 4: Oil-Red-O staining of GFP+ MSC in passage 5 after differentiation into adipocytes.

Results: MSCs from WKY wildtype as well as from WKY-GFP+ rats could be successfully differentiated in culture. They were shown to be plastic adherent, to express CD90 and to lack expression of CD45 and CD34. Only a minority of the cells was positive for CD44 (Fig. 1, Fig. 2). Furthermore, the cells could be differentiated into adipocytes and osteocytes (Fig. 3, Fig. 4). Interestingly, the expression of GFP in cells from GFP+ rats was strong, and green fluorescence was present up to passage 10. In our analysis of the effects of the different supernatants on Mrc1 expression levels of macrophages, supernatants coming from GFP+ MSCs were shown to downregulate Mrc1 expressions more effectively than supernatants coming from WT MSCs. This was significant (P00.03) for unstimulated macrophges (Fig. $5 \mathrm{a}+\mathrm{b}$ ) There was no significant difference in antiinflammatory potential of supernatants coming from MSCs from older donors compared to younger donors (Fig. $5 \mathrm{c}+\mathrm{d}$ ).
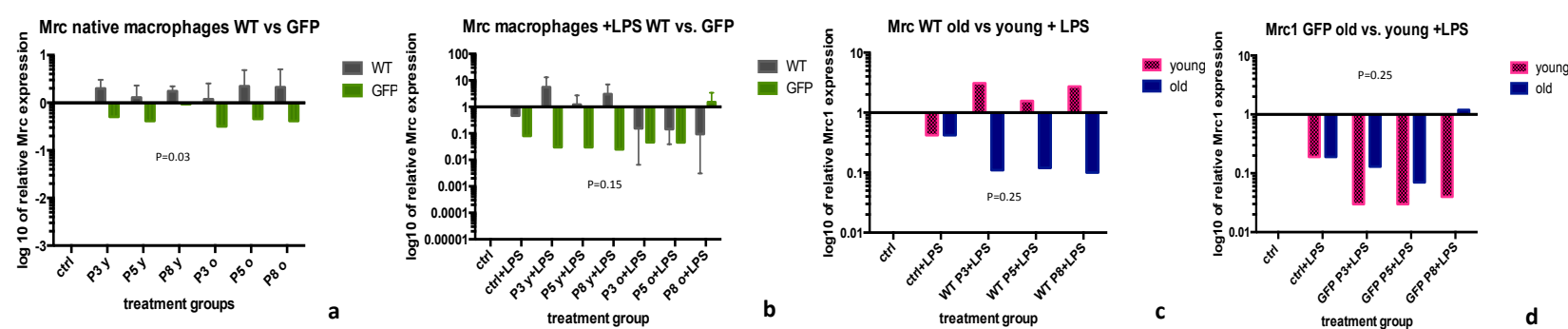

Fig.5. the graph shows the influence of different MSC culture supernatants on the expressions levels of Mrc1 on bone marrow derived macrophages (BMDM) coming from a WKY-WT rat. Supernatants coming from GFP+ MSCs showed a stronger downregulation of Mrc1 than supernatants coming from WT cells (a, b). There was no siginificant difference between the action of supernatants from MSCs coming from older donors compared to young donor animals, however supernatants from young WT caused an upregulation of Mrc1 and are therefore the most promising, antiinflammatory agents (c,d).

Conclusion: MSCs from transgenic WKY rats positive for the expression of GFP show strong green fluorescence throughout passages 0-10. Supernatants coming from WT-MSCs had upregulatory effect on Mrc1 expressions of macrophages and could even reverse the inflammatory stimulus of LPS treatment. This experiment indicates, that there is differences between WT and GFP MSCs concerning their paracrine actions. Further experiments have to clarify, whether there is differences in direct actions of the cells on inflammation via cell-cell contact.

Referneces:

1.Ali F, Dua A, Cronin DC. Changing paradigms in organ preservation and resuscitation. Curr Opin Organ Transplant [Internet]. 2015 Apr [cited 2015 Jul 3];20(2):152-8.

2.Van Raemdonck D, Neyrinck A, Rega F, Devos T, Pirenne J. Machine perfusion in organ transplantation: a tool for ex-vivo graft conditioning with mesenchymal stem cells? Curr. Opin. Organ Transplant. 2013;18(1):24-33 